\title{
Atomic layer stacking structure and negative uniaxial magnetocrystalline anisotropy of $\mathrm{Co}_{100-x} \mathrm{Ir}_{x}$ sputtered films
}

\author{
N. Nozawa, S. Saito, T. Kimura, K. Shibuya*, K. Hoshino*, \\ S. Hinata, and M. Takahashi** \\ Department of Electronic Engineering, Graduate School of Engineering, Tohoku University, 6-6-05 Aoba, Aramaki, Aoba-ku, \\ Sendai 980-8579, Japan \\ * Foundation for Promotion of Material Science and Technology of Japan, 1-18-6 Kitami, Setagaya-ku, Tokyo 157-0067, Japan \\ ** New Industry Creation Hatchery Center, Tohoku University, 6-6-10 Aoba, Aramaki, Aoba-ku, Sendai 980-8579, Japan
}

The negative uniaxial magnetocrystalline anisotropy $\left(K_{\mathrm{u}}\right)$ was evaluated for various compositions of Co100-X $\mathrm{Ir}_{X}$ thin films with respect to the atomic layer stacking structure. Pure Co film fabricated at a substrate temperature $\left(T_{\text {sub }}\right)$ of $600{ }^{\circ} \mathrm{C}$ was found to have a positive $K_{\mathrm{u}}$ of $6.1 \times 10^{6} \mathrm{erg} / \mathrm{cm}^{3}$. With increasing $x$, the sign of $K_{\mathrm{u}}$ changed from positive to negative, and the negative $K_{\mathrm{u}}$ took a maximum value of $-9.6 \times 10^{6} \mathrm{erg} / \mathrm{cm}^{3}$ at around $x=20$ at. \% for films fabricated at $T_{\mathrm{sub}}=600{ }^{\circ} \mathrm{C}$. Adding more Ir decreased the absolute value of the negative $K_{\mathrm{u}}$ which became 0 over $x=$ 50 at. \%. X-ray diffraction analysis and scanning transmission electron microscopy revealed that the atomic layer stacking structure of the $\mathrm{Co}_{100-X} \mathrm{Ir}_{x}$ films changed from $-\mathrm{A}-\mathrm{B}-\mathrm{A}-\mathrm{B}-(\mathrm{hcp})$ to $-\mathrm{A}-\mathrm{B}-\mathrm{C}-\mathrm{A}-\mathrm{B}-\mathrm{C}-$ (fcc) stacking with increasing Ir content. Moreover, Coso $\mathrm{Ir}_{20}$ grains were revealed to consist of 2 kinds of randomly located composition-modulated atomic layers, nearly pure-Co and pure-Ir layers, while Co and Co50Ir50 had disordered structures. In this paper, a new perspective on the atomic layered structure with superlattice diffraction, which is different from the conventional "ordered structure", is discussed.

Key words: Negative uniaxial magnetocrystalline anisotropy, CoIr sputtered film, Superlattice line, High-angle annular dark-field, Stacking faults

\section{Introduction}

Recently, much attention has been paid to materials with negative uniaxial magnetocrystalline anisotropy $\left(K_{\mathrm{u}}\right)$, with a hard magnetized axis and an easy magnetized plane, because of their potential for use in many anticipated applications. For example, by using a negative $K_{\mathrm{u}}$ material as a soft magnetic underlayer for perpendicular magnetic recording media, the spike noise and wide adjacent track erasure can be reduced. ${ }^{\left.1{ }^{1,2}\right)} \mathrm{A}$ positive-/ negative- $K_{\mathrm{u}}$ exchange coupled recording media decrease the switching field through the local spin twisted structure. ${ }^{3), 4)}$ The field generation layer (FGL) of a spin torque oscillator with negative $K_{\mathrm{u}}$ can realize high oscillation frequency because the magnetization of FGL becomes stable in the plane direction. ${ }^{5), 6)}$ As for the materials, $\quad \alpha-\mathrm{Fe}-\mathrm{C},{ }^{7)} \quad$ dhcp-CoFe, ${ }^{8} \quad$ NiAs-type $\mathrm{Mn}_{50} \mathrm{Sb}_{50},{ }^{9)}$ and disordered hcp-CoIr ${ }^{10)}$ are all known to exhibit negative $K_{\mathrm{u}}$ at room temperature (RT). In particular, the disordered hcp-CoIr alloy is considered to be useful because this alloy shows negative $K_{\mathrm{u}}$ for a wide range of Ir content and is in a thermal equilibrium phase at RT. According to previous reports on hcp-CoIr alloy films with $c$-plane sheet texture, the maximum value of the negative $K_{\mathrm{u}}$ was $-6.0 \times 10^{6} \mathrm{erg} / \mathrm{cm}^{3}$ at around $\mathrm{Ir}$ content of 20 at. \% even in a disordered structure. ${ }^{1,2), 11)}$

To further increase the negative $K_{\mathrm{u}}$, the following approaches are thought to be effective with the intention to increase spin-orbital interaction: 1) perfect -A-B-A-B- (hcp) stacking without stacking faults (SFs), and 2) atomic site ordering in the alloy. As an example of 1), reducing the probability of fcc stacking
(-A-B-C-A-B-C- stacking) from $10 \%$ to $0 \%$ was found to yield a 2.3 times larger $K_{\mathrm{u}}$ for a pure Co film. ${ }^{12)}$ As for an example of 2), structural change from a disordered structure to ordered $\mathrm{m}-\mathrm{D}_{19} \mathrm{Co}_{75} \mathrm{Pt}_{25}$ increased $K_{\mathrm{u}}$ to a 7.5 times larger value. ${ }^{13)}$ In general, in order to induce regularity of the atomic layer sequence and of the atomic site arrangement, promotion of atomic migration during sputter deposition is considered to be effective. In this paper, the dependence of the magnetic properties and atomic layer stacking structure on the Ir content are evaluated for CoIr films fabricated by a substrate heating process, and correlation between negative $K_{\mathrm{u}}$ and the structure is discussed in relation to SFs and atomic site arrangement.

\section{Experimental procedure}

All samples prepared in the present study were fabricated by direct-current (dc) magnetron sputtering onto 2.5-in. crystallized glass substrates. The layered structure of the sample was substrate/Ta $(10 \mathrm{~nm}) / \mathrm{Pt}(10$ $\mathrm{nm}) / \mathrm{Ru} \quad(20 \mathrm{~nm}) / \mathrm{Co}_{100-x} \mathrm{Ir}_{x}(20 \mathrm{~nm}) / \mathrm{C} \quad(7 \mathrm{~nm})$. The thickness combination of the $\mathrm{Ta} / \mathrm{Pt} / \mathrm{Ru}$ underlayer was optimized to enhance the negative $K_{\mathrm{u}}$ for a $\mathrm{Co}_{80} \mathrm{Ir}_{20}$ film. ${ }^{14)}$ First, the underlayer was deposited under an $\mathrm{Ar}$ gas pressure of $0.6 \mathrm{~Pa}$ at $\mathrm{RT}$ and heated up to $700{ }^{\circ} \mathrm{C}$, for the completion of structural relaxation inside the underlayer. Then, the underlayer was cooled in the vacuum chamber and the Co100-X $\mathrm{Ir}_{x}$ film was deposited on the underlayer under an Ar gas pressure of 3.0 Pa (the optimum Ar gas pressure to increase the intensity of superlattice diffraction) at the desired substrate 
temperature, $T_{\text {sub. In our previous report, }} T_{\text {sub }}$ of $600{ }^{\circ} \mathrm{C}$ was found to be effective for enhancement of negative $K_{\mathrm{u}}$ of the $\mathrm{Co}_{80} \mathrm{Ir}_{20}$ film. ${ }^{14)}$ Therefore, $600{ }^{\circ} \mathrm{C}$ and RT (as a reference) were selected for $T_{\text {sub }}$ in this study. The Co100-X $\operatorname{Ir}_{x}$ films were deposited by co-sputtering with Co and Ir targets by varying the discharge power for each target. All of the $\mathrm{Co}_{100-x} \mathrm{Ir}_{x}$ films in the present study were confirmed to be $c$-plane oriented [see Fig. 2 and Fig. 4]. The magnetic properties were measured using a vibrating sample magnetometer and a torque magnetometer. The torque curves were measured in various fields up to $25 \mathrm{kOe}$ and Fourier analysis was applied. Accounting for the self-demagnetizing energy, $K$ u for $c$-plane oriented films can be expressed as:

$$
K_{\mathrm{u}}=L_{2 \theta}^{\mathrm{H} \rightarrow \infty}+2 \pi M_{\mathrm{s}}^{2},
$$

where $M_{\mathrm{s}}$ represents the saturation magnetization, $L_{2 \theta}{ }^{\mathrm{H} \rightarrow \infty}$ is the torque coefficient of the twofold component at $H \rightarrow \infty$, which is obtained by extrapolating the coefficient versus $1 / H$ curve to $1 / H \rightarrow 0$ in the range from $17 \mathrm{kOe}$ to $25 \mathrm{kOe}$ [See Fig. 1(a)]. Although the applied field of $25 \mathrm{kOe}$ is not sufficient to saturate the magnetization along the field direction for CoIr films, $L_{2 \theta}{ }^{\mathrm{H} \rightarrow \infty}$ is confirmed to be nearly the same as the saturation torque coefficient $\left(L_{2 \theta}\right.$ sat) derived from a simulation assuming a single-domain state [See Fig. 1(b)]. The magnitude of $K_{\mathrm{u}}$ derived from Eq. (1) was supported by the result from $\mathrm{X}-$ and Q-band ferromagnetic resonance measurements through the uniaxial magnetic anisotropy field, $H_{\mathrm{k}}\left(H_{\mathrm{k}}=2 K_{\mathrm{u}} / M_{\mathrm{s}}\right)$. For the analysis of the atomic layered stacking structure,

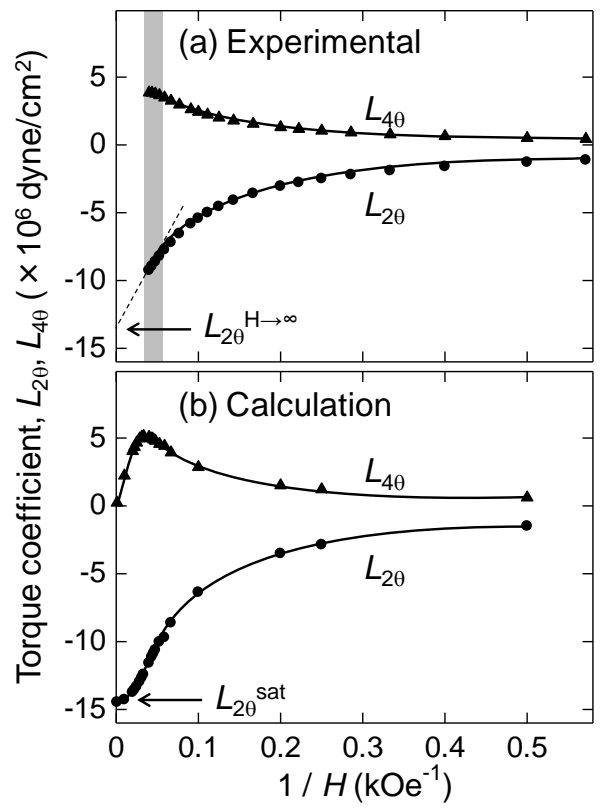

Fig. 1 The torque coefficients of the twofold and fourfold components, $L_{2_{\theta}}$ and $L_{4_{\theta}}$, as a function of the reciprocal of the applied field $1 / H$ for the $\mathrm{Co}_{80} \mathrm{Ir}_{20}$ film $\left(M_{\mathrm{s}}=908 \mathrm{emu} / \mathrm{cm}^{3}, K_{\mathrm{u}}=-9.28 \times 10^{6} \mathrm{erg} / \mathrm{cm}^{3}\right)$ as a typical example. (a) Experimental and (b) calculation results. The gray region in (a) is the $1 / H$ range used for extrapolating $L_{2 \theta}{ }^{\mathrm{H} \rightarrow \infty}$. For (b), calculation was conducted by assuming a single-domain state.
$\mathrm{X}$-ray diffraction (XRD) and transmission electron microscopy (TEM) were carried out. For the XRD analysis, out-of-plane and in-plane diffraction profiles were measured using $\mathrm{Cu} K \alpha$ radiation. The incident angle of X-rays for in-plane XRD was $0.4^{\circ}$, so that the profiles reflected structural information at a depth of approximately $20 \mathrm{~nm}$ from the surface. In the TEM analysis, the high-angle annular dark-field (HAADF) method with a detection angle of $40-210^{\circ}$ was adopted by scanning with an electron beam probe of $0.1 \mathrm{~nm}$ diameter (STEM).

\section{Results and Discussion}

\subsection{Magnetic properties}

Fig. 2 shows (a) $M_{\mathrm{s}}$ and (b) $K_{\mathrm{u}}$ for the $\mathrm{Co}_{100_{-} \mathrm{I}} \mathrm{Ir}_{X}$ films as a function of the Ir content, $x$. The solid circles indicate the samples deposited under a substrate temperature of $600{ }^{\circ} \mathrm{C}$. Open triangles are at RT as a reference. According to Fig. 2(a), $M_{\mathrm{s}}$ gradually decreased with an increase in $x$ and was independent of $T_{\text {sub. On }}$ the other hand, the magnitude of $K_{\mathrm{u}}$ drastically changed as a function of both $x$ and $T_{\text {sub. For the films with } x=0}$ at. \% (pure Co film), the $K_{\mathrm{u}}$ value increased to $6.1 \times 10^{6}$ $\mathrm{erg} / \mathrm{cm}^{3}$ at $T_{\text {sub }}=600{ }^{\circ} \mathrm{C}$, which is the same as that for hcp Co bulk, from $1.9 \times 10^{6} \mathrm{erg} / \mathrm{cm}^{3}$ at $T_{\text {sub }}=\mathrm{RT}$. With increasing $x$, the sign of $K_{\mathrm{u}}$ changed from positive to negative, and negative $K_{\mathrm{u}}$ took a maximum value of $-9.6 \times 10^{6} \mathrm{erg} / \mathrm{cm}^{3}$ at around $x=20$ at. $\%$ for the film at $600{ }^{\circ} \mathrm{C}$. Due to the substrate heating process, the maximum negative $K_{\mathrm{u}}$ at $T_{\mathrm{sub}}=600^{\circ} \mathrm{C}$ was found to be enhanced to 1.4 times the value at $\mathrm{RT}$.

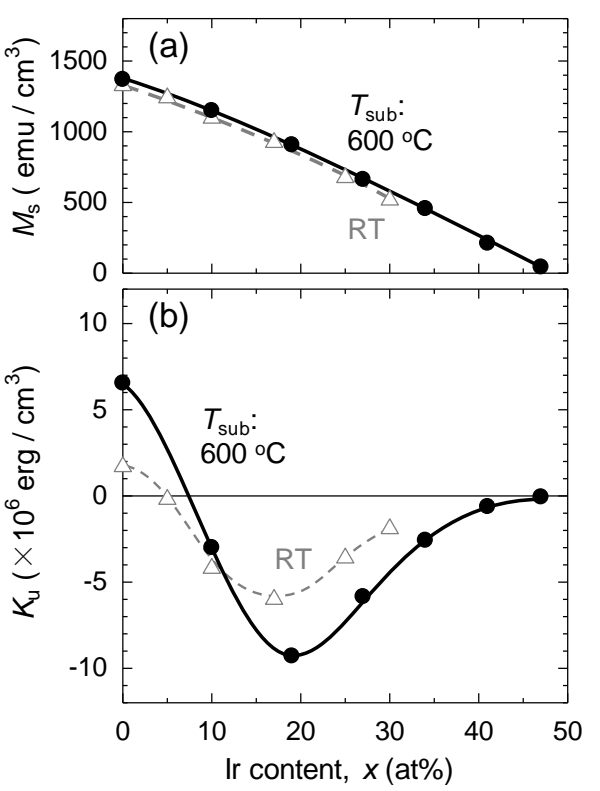

Fig. 2 Dependence of (a) saturation magnetization $M_{\mathrm{s}}$ and (b) uniaxial magnetocrystalline anisotropy $K_{\mathrm{u}}$ on Ir content $x$ for the $\mathrm{Co100-X}_{-} \mathrm{Ir}_{x}$ films. The layered structure of the samples is sub./Ta (10)/Pt (10)/Ru (20)/CoIr (20). Black circles and gray triangles indicate samples deposited under a substrate temperature of $600{ }^{\circ} \mathrm{C}$ and $\mathrm{RT}$, respectively. 


\subsection{Atomic layer stacking sequence}

Fig. 3 shows in-plane XRD profiles for $\mathrm{Co}_{100-x} \mathrm{Ir}_{x}$ films at $T_{\text {sub }}=600{ }^{\circ} \mathrm{C}$ for various Ir content as a typical example. In the pure Co film, diffraction peaks at $2 \theta_{\chi} \sim$ $41.5^{\circ}$ and $75.7^{\circ}$ can be observed and were identified as hcp (10.0) and (11.0), respectively. With increasing Ir content, the (11.0) diffraction peak smoothly shifted toward the lower angle side. Therefore, the formation of the $c$-plane sheet texture and solid solution were confirmed for all $\mathrm{Co}_{100-X} \mathrm{Ir}_{X}$ films. In the case of the (10.0) diffraction, strong intensities can be observed in the films with $x=0-35$ at. \% and the intensity decreased with more Ir content, and disappeared over 53 at. \%. This means that the degree of SFs induced into magnetic grains is considered to change as a function of $\mathrm{Ir}$ content. ${ }^{15-17)}$ Therefore, quantitative evaluation of SFs was carried out.

Fig. 4 shows the integral intensity ratio between (10.0) and (11.0) diffractions corrected by the Lorentz-polarization factor and atomic scattering factor (corrected $I_{10.0} / I_{11.0}$ ) as a function of Ir content $x$. Solid circles and open triangles indicate the samples deposited under $T_{\text {sub }}$ of $600{ }^{\circ} \mathrm{C}$ and RT, respectively. Here, the corrected $I_{10.0} / I_{11.0}$ means the index of the degree of SFs induced into hcp and/or fcc grains quantitatively. ${ }^{15}$ ) Theoretical values of the corrected $I_{10.0} / I_{11.0}$ are 0 for perfect fcc stacking and 0.25 for perfect hcp stacking. As for the $\mathrm{Co}_{100-X} \mathrm{Ir}_{X}$ films at $T_{\text {sub }}=\mathrm{RT}$ in Fig. 4, the Co film showed the corrected $I_{10.0} / I_{11.0}$ of about 0.05 , which indicated that the probability of fcc stacking appearance $\left(P_{\text {fcc }}\right)$ was $10 \%$. With increasing Ir content, the corrected $I_{10.0} / I_{11.0}$ had a broad peak at around 30 at. \% and had a maximum value of 0.20 . On the other hand, focusing on the films at $600{ }^{\circ} \mathrm{C}$, even the Co film showed the corrected $I_{10.0} / I_{11.0}$ of about 0.20 , which corresponds to $P_{\text {fcc }}=0.05 \%$, nearly perfect hcp stacking. The corrected $I_{10.0} / I_{11.0}$ increased with increasing Ir content and had a maximum at 19 at. \%. With more Ir content, the corrected $I_{10.0} / I_{11.0}$ decreased drastically and became 0

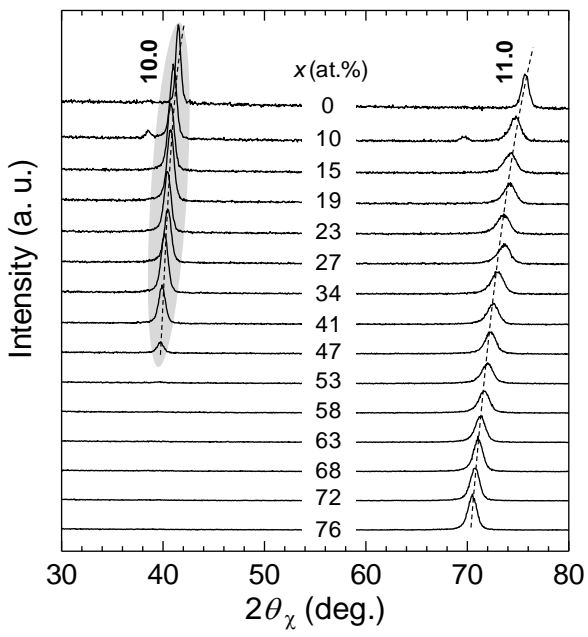

Fig. 3 In-plane $\mathrm{x}$-ray diffraction profiles for sub./Ta (10)/Pt (10)/Ru (20)/Co100-X $\operatorname{Ir}_{X}$ (20) films deposited at $T_{\text {sub }}$ $=600{ }^{\circ} \mathrm{C}$. The top profile is for the Co film and the ones below are for films with increased Ir content. over 47 at. $\%$. Note that in the region of $x=15-23$ at. $\%$, the value of the corrected $I_{10.0} / I_{11.0}$ for the films deposited at $600{ }^{\circ} \mathrm{C}$ exceeded the theoretical limit of 0.25 . Therefore, in order to reveal the reason for this excess, detailed analysis of the atomic layer stacking was carried out.

\subsection{Composition-modulated atomic layer stacking}

Fig. 5 shows out-of-plane XRD profiles for the Co100- $\operatorname{Ir}_{x}$ films fabricated at $T_{\text {sub }}=600{ }^{\circ} \mathrm{C}$ in the Bragg angle range of (a) $17-23^{\circ}$ and (b) $37-45^{\circ}$. In Fig. 5(a), a diffraction peak at $2 \theta \sim 44.5^{\circ}$ can be observed for the pure Co film, which is identified as hcp (00.2), and it smoothly shifted to the lower side with an increase of Ir content, similar to the in-plane XRD profiles. Note that diffraction at $2 \theta \sim 21.5^{\circ}$ can be observed uniquely for films with $x=10-34$ at. \%. Judging from the lattice spacing of the (00.2) plane, this diffraction peak is identified as hcp (00.1), which means the superlattice line.

In order to directly observe the superlattice structure, we used STEM analysis. In our previous study, the HAADF method was found to be quite effective to observe layers sequence and compositional atomic layer stacking structure. ${ }^{14)}$ The intensity of a HAADF image is generally proportional to the square of the average atomic number of a sample. $\left.{ }^{18)}, 19\right)$ Therefore, the contrast of the HAADF image is mainly caused by the difference in composition. Fig. 6 shows cross-sectional STEM images using the HAADF detector for (a) Co, (b) $\mathrm{Co}_{81} \mathrm{Ir}_{19}$, and (c) $\mathrm{Co}_{47} \mathrm{Ir}_{53}$ films deposited at $T_{\text {sub }}=600{ }^{\circ} \mathrm{C}$, respectively. The left and right images show a wide-field and enlarged views of the $\mathrm{Co100}_{-} \mathrm{Ir}_{x}$ films, respectively. The up and down directions in the views correspond to the film normal direction. The $\mathrm{Co}_{100-} \mathrm{Ir}_{X}$ grains with the [11.0] direction which were parallel to the incident direction of the electron beam were selected for observation. According to the right image in Fig. 6(a), many scattered dots can be seen which correspond to Co or Ir atoms. With respect

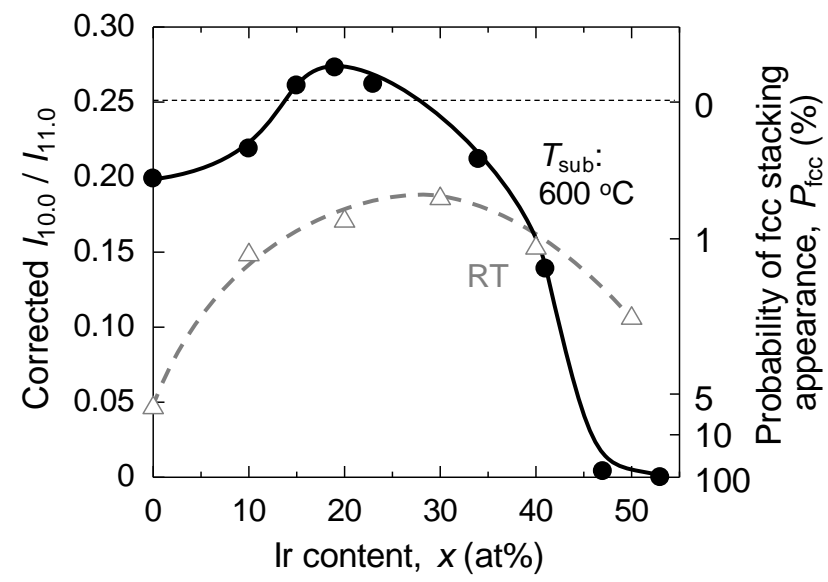

Fig. 4 Integral intensity ratio between (10.0) and (11.0) diffractions corrected by the Lorentz-polarization factor and atomic scattering factor (Corrected $I_{10.0} / I_{11.0}$ ) as a function of Ir content $x$. Solid circles and open triangles indicate samples deposited under $T_{\text {sub }}$ of $600{ }^{\circ} \mathrm{C}$ and RT, respectively. The right vertical axis means the probability of fcc stacking appearance. 
to the atomic arrangements, some atoms were found to be aligned in a straight line at an angle of $20^{\circ}$ from the film normal while other atoms were arranged in a zigzag line. In these HAADF images, the dots arranged in a straight line and in a zigzag line mean fcc stacking (-A-B-C-A-B-C- stacking) and hep stacking (-A-B-A-B- stacking), respectively. Hence, the stacking structure of the Co films fabricated at $600{ }^{\circ} \mathrm{C}$ is thought to be hcp stacking with a small amount of fcc stacking. As for the $\mathrm{Co}_{47} \mathrm{Ir}_{53}$ film in Fig. 6(c), all of the atoms are aligned in a straight line with a slope of $20^{\circ}$ and thus found to have nearly perfect fcc stacking. In contrast, all the atoms were arranged into a zigzag manner in the $\mathrm{Co}_{81} \mathrm{Ir}_{19}$ film in Fig. 6(b), which means nearly perfect hcp stacking. Next, focusing on the brightness of the dots in the HAADF images, all of the dots in the Co and $\mathrm{Co}_{47} \mathrm{Ir}_{53}$ film showed the same brightness in Fig. 6(a) and (c). On the other hand, in the $\mathrm{Co}_{81} \mathrm{Ir}_{19}$ film, 2 kinds of dots, bright and dark, were observed as shown in Fig. 6(b). The bright dots were found to be aligned along some horizontal lines parallel to the film plane and randomly located into dark horizontal lines consisting of dark dots. It can be easily understood that the bright and dark lines indicate layers which contain more Ir atoms (Ir-rich layers) and less Ir atoms (Ir-poor layers), respectively. Thus, the $\mathrm{Co}_{11} \mathrm{Ir}_{19}$ film consisted of composition-modulated atomic layers. Judging from Fig. 6(b), $10 \mathrm{Ir}$-rich layers were formed among a total of 53 layers, as shown by the arrows. In other words, the probability of Ir-rich layers is $20 \%$, which corresponds to the composition of this film.

In order to consider the compositional modulation in Co81 Ir 19, the possible compositional pair for 2 atomic layers was calculated. When the compositions of 2 atomic layers are defined as $\mathrm{Co}_{100-y} \mathrm{Ir}_{y}$ (Ir-rich layer) and Co100-Z $\operatorname{Ir}_{Z}$ (Ir-poor layer), and the existence probabilities

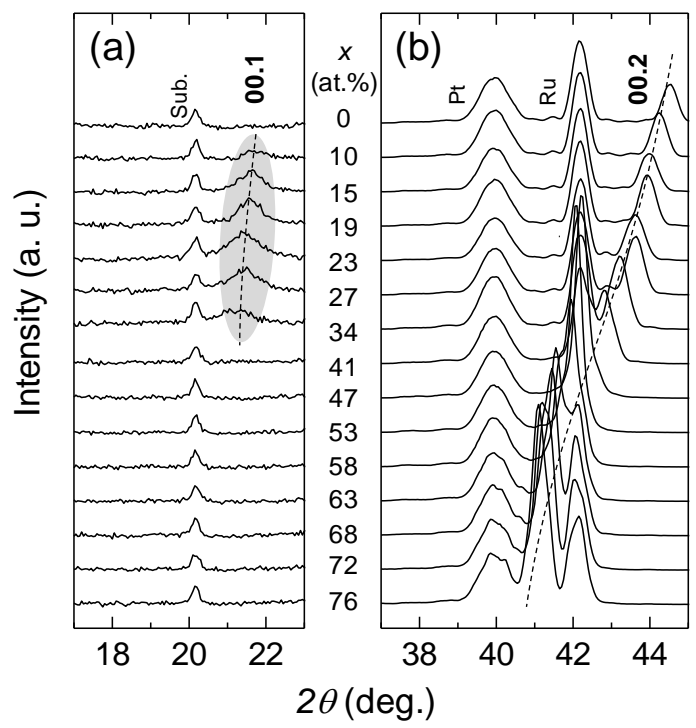

Fig. 5 Out-of-plane $\mathrm{x}$-ray diffraction profiles for sub./Ta $\quad(10) / \mathrm{Pt} \quad(10) / \mathrm{Ru} \quad(20) / \mathrm{Co}_{100-{ }_{X} \mathrm{Ir}_{x}} \quad$ (20) films deposited at $T_{\mathrm{sub}}=600{ }^{\circ} \mathrm{C}$ in the Bragg angle range of (a) $17-23^{\circ}$ and (b) $36-45^{\circ}$. The top profile is for the Co film and ones below are for the films with increased Ir content. of Ir-rich and Ir-poor layers are also defined as $p(\%)$ and $100-p(\%)$, respectively, film composition ratio can then be expressed as:

$$
\begin{aligned}
\mathrm{Co}: \operatorname{Ir} & =\frac{p(100-y)+(100-p)(100-z)}{100}: \frac{p y+(100-p) z}{100} \\
& (y \geq z) .
\end{aligned}
$$

For the $\mathrm{Co}_{80} \mathrm{Ir}_{20}$ film, the value of $p$ was revealed to be $20 \%$ from Fig. 6(b), and thus the Ir content in the film is expressed as:

$$
\begin{aligned}
& \text { (Ir content in the film })=20=\frac{20 y+80 z}{100}, \\
& \therefore y+4 z=100(y \geq z) .
\end{aligned}
$$

Therefore, the composition of the Ir-rich layer was found to have a linear dependence on that of the Ir-poor layer. Fig. 7 shows the relation between the Ir content in the Ir-rich and Ir-poor layers given by Eq. 3. The maximum value of $z$ is $20 \%$ ( $\mathrm{Ir}-$ poor: $\mathrm{Cos}_{0} \mathrm{Ir}_{20}$ ) when the value of $y$ is $20 \%$ (Ir-rich: $\mathrm{Co}_{80} \operatorname{Ir}_{20}$ ), which means a disordered structure. With increasing $y$, the composition difference

(a)

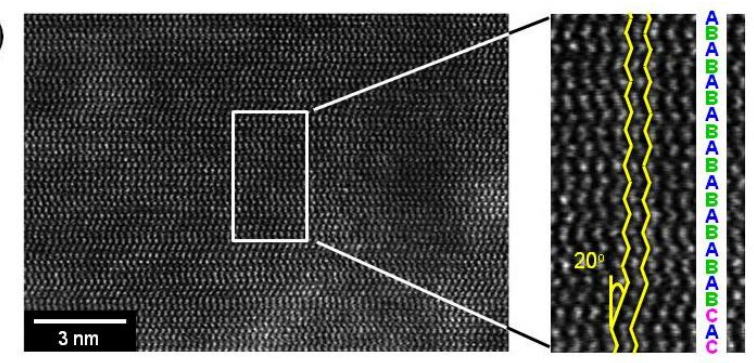

(b)

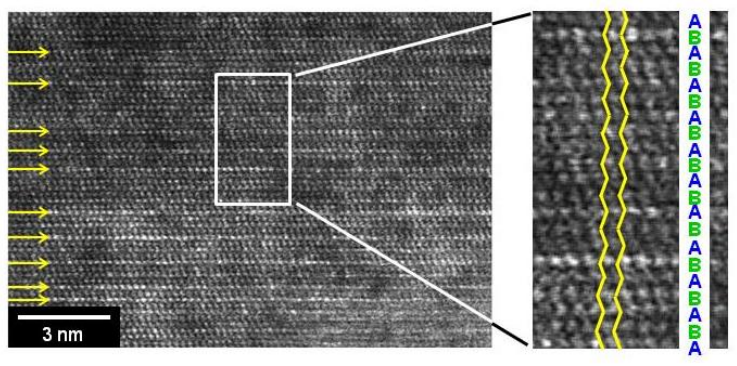

(c)

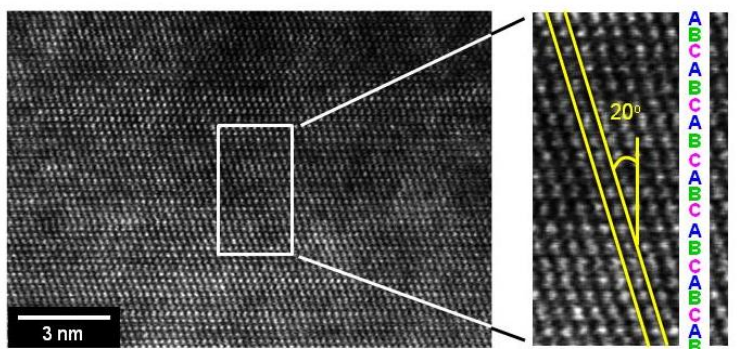

Fig. 6 Cross-sectional high-angle annular dark-field images obtained by scanning transmission electron microscopy (STEM) for (a) Co, (b) Co81 Ir 19 , and (c) $\mathrm{Co}_{47} \operatorname{Ir}_{53}$ films deposited at $T_{\text {sub }}=600{ }^{\circ} \mathrm{C}$. Left and right images show wide-field and enlarged views of the Co100- $\operatorname{Ir}_{x}$ films. In the right images, the stacking layers were identified as the A, B, and C atomic layers. Arrows in the left (b) indicate bright dots aligned as a horizontal line. 
between the Ir-rich and Ir-poor layers becomes conspicuous and reaches $100 \%$, which indicates a stacking structure with pure-Co and pure-Ir layers. Although there are numerous variations in the possible compositional pair for the Ir-rich and Ir-poor layers expressed by Eq. (3), clear contrast of the HAADF image between bright and dark layers suggests that nearly pure-Co layers and pure-Ir layers tend to form in the hcp stacking concerning the existence ratio of bright layers.

\subsection{Proposed atomic layer stacking structure with superlattice diffraction}

Based on these results, we summarized the (a) conventional and (b) proposed models of the atomic layer stacking structure for the CoIr grains with superlattice diffraction as shown in Fig. 8. According to previous reports on Co-based alloy films, the (00.1) diffraction is always discussed in relation to the formation of an ordered structure consisting of 2 different composition layers deposited alternately as in Fig. 8(a).13),20),21) However, the present results introduced in this paper indicate a new atomic layer stacking structure consisting of randomly sequenced nearly pure-Ir and pure-Co layers, as shown in Fig. 8(b). Our proposed composition-modulated atomic layer stacking structure gives a new perspective on the so-called "ordered structure" with superlattice diffraction. The formation of this structure with hcp stacking is consistent with the appearance of superlattice diffraction in Fig. 5(a).

It is known that the integrated intensity of the diffraction is proportional to the squared structure factor $|F|^{2}{ }^{22)}$ If a unit cell contains atoms $1, \cdots, N$, with fractional coordinates $u_{1} v_{1} W_{1}, \cdots, u_{N} v_{N} W_{N}$, and atomic scattering factors $f_{1}^{\text {hk.l }}, \cdots, f_{N}^{\text {hk.l }}$, then the structure factor for the $h k .1$ reflection is given by:

$$
F_{h k . l}=\sum_{j=1}^{N} f_{j}^{h k . l} e^{-2 \pi i(h k . l) \cdot\left(u_{j} v_{j} \cdot w_{j}\right)} .
$$

$F_{00.1}$ with 1 of $N / 2$ for Co-Ir alloys, which corresponds to superlattice diffraction, can be expressed as:

$$
\begin{aligned}
F_{00 . N / 2} & =f_{\mathrm{Co}}^{00 . N / 2} \sum_{j} e^{-2 \pi i \cdot w_{j} \frac{N}{2}}+f_{\mathrm{Ir}}^{00 . N / 2} \sum_{j} e^{-2 \pi \cdot w_{j} \frac{N}{2}} \\
& =\left(N_{\text {Even }}^{\mathrm{Co}}-N_{\text {Odd }}^{\mathrm{Co}}\right) f_{\mathrm{Co}}^{00 . N / 2}+\left(N_{\text {Even }}^{\mathrm{Ir}}-N_{\text {Odd }}^{\mathrm{Ir}}\right) f_{\mathrm{Ir}}^{00 . N / 2},
\end{aligned}
$$

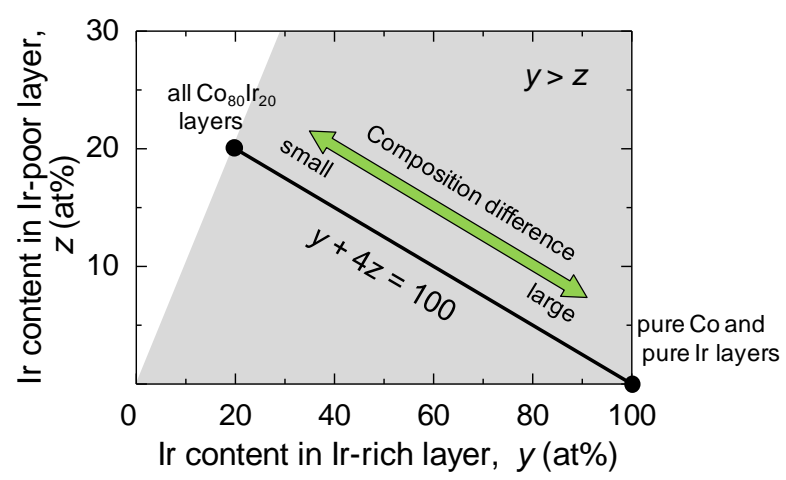

Fig. 7 Compositional difference between two kinds of atomic layers, Co100-y $\operatorname{Ir}_{y}$ (Ir-rich layer) and $\mathrm{Co}_{100-z} \mathrm{Ir}_{z}$ (Ir-poor layers), in the Coso $\operatorname{Ir}_{20}$ alloy film. where $N_{\text {Even }}{ }^{\text {Co }}$ and $N_{\text {Odd }}{ }^{\text {Co }}$ represent the number of Co, and $N_{\text {Even }}{ }^{\text {Ir }}$ and $N_{\text {Odd }}{ }^{\text {Ir }}$ indicate the number of Ir atoms which appeared in even- and odd-number layers in the unit cell, respectively. $f_{\mathrm{CO}_{0}}{ }^{00 . N / 2}$ and $f_{\mathrm{r}}{ }^{00 . N / 2}$ represent the scattering factors for $\mathrm{Co}$ and $\mathrm{Ir}$ atoms.For the Co80 Ir20 alloy with a total of 100 atomic layers (about $20 \mathrm{~nm}$ thickness) with perfect hcp stacking, $N_{\text {Even }}{ }^{\text {Co or Ir }}$ and $N_{\text {Odd }}{ }^{\mathrm{Co}}$ or Ir correspond to $N_{\mathrm{A}}$ Co or $\mathrm{Ir}$ and $N_{\mathrm{B}}$ Co or Ir, respectively. Therfore, the following 4 equations are given:

$$
\begin{aligned}
& N_{\mathrm{A}}^{\mathrm{Co}}+N_{\mathrm{B}}^{\mathrm{Co}}=80, \quad N_{\mathrm{A}}^{\mathrm{Ir}}+N_{\mathrm{B}}^{\mathrm{Ir}}=20, \\
& N_{\mathrm{A}}^{\mathrm{Co}}+N_{\mathrm{A}}^{\mathrm{Ir}}=50, \quad N_{\mathrm{B}}^{\mathrm{Co}}+N_{\mathrm{B}}^{\mathrm{Ir}}=50 .
\end{aligned}
$$

Using these equations, $F_{00 . N 2}$ for the $\mathrm{C}_{80} \mathrm{Ir}_{20}$ alloy can be simply written as:

$$
F_{00 . N / 2}=\left(f_{\mathrm{Ir}}-f_{\mathrm{Co}}\right)\left(2 N_{\mathrm{A}}^{\mathrm{Ir}}-20\right) .
$$

Therefore, the appearance of the superlattice diffraction from each grain depends on $N_{\mathrm{A}}{ }^{\mathrm{Ir}}$.

Next, when 20 Ir layers are randomly formed in a total of 100 layers, the probability of appearance of a grain $\left(P_{\text {grain }}\right)$ with a certain $N_{\mathrm{A}}^{\mathrm{Ir}}$ can be expressed as:

$$
P_{\text {grain }}=\frac{{ }_{50} C_{N_{\mathrm{A}}^{\mathrm{Ir}}} \times{ }_{50} C_{20-N_{\mathrm{A}}^{\mathrm{Ir}}}}{{ }_{100} C_{20}} .
$$

Fig. 9 shows the dependence of $P_{\text {grain }}$ on $N_{\mathrm{A}}$ Ir for the hcp $\mathrm{Co}_{80} \mathrm{Ir}_{20}$ film with composition-modulated atomic layer (a)

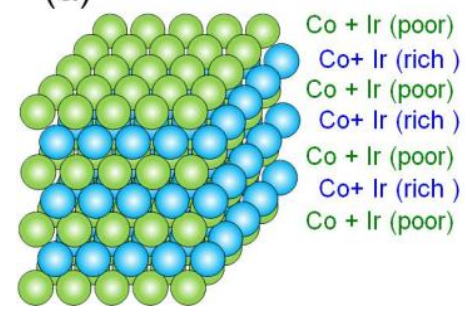

(b)

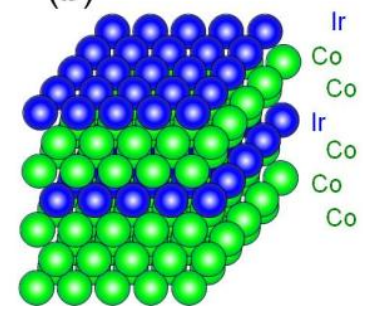

Fig. 8 (a) Conventional and (b) proposed stacking structure models for $\mathrm{Co}-\mathrm{Ir}$ grains with superlattice diffraction.

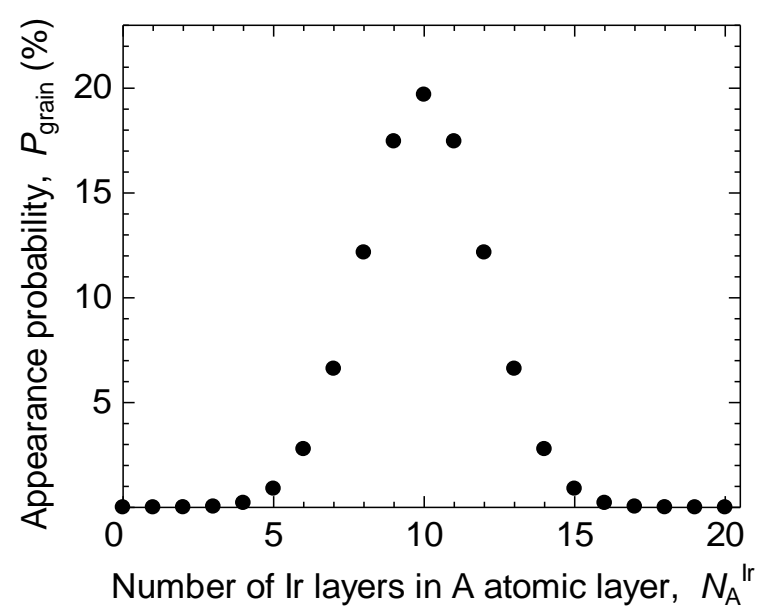

Fig. 9 Dependence of appearance probability of the grain $\left(P_{\text {grain }}\right)$ on $N_{\mathrm{A}}$ Ir for the hcp Co80 $\operatorname{Ir}_{20}$ film with composition-modulated atomic layer stacking. 
stacking. The grains containing equal numbers of Ir layers in $\mathrm{A}$ and $\mathrm{B}$ of the $-\mathrm{A}-\mathrm{B}-\mathrm{A}-\mathrm{B}-$ atomic layer stacking $\left(N_{\mathrm{A}} \mathrm{Ir}=10\right)$ were found to have the highest $P_{\text {grain }}$ value. The shape of curve seems to be a Gaussian distribution. Based on this result, the relationship between $P_{\text {grain }}$ and $F_{00 . N / 2} / F_{00 . N 22^{\text {max }}}$ is given in Fig. 10 . The appearance probability of the grains with $F_{00 . N / 2} / F_{00 . N 22^{\max }}=0$ is the highest, but that with $F_{00 . N / 2} / F_{00 . N 22^{\max }} \neq 0$ is not 0 . Therefore, statistically, both grains exist in the film and the superlattice diffraction from the film appears because diffracted X-rays from different grains do not interfere.

\subsection{Corrected intensity ratio for composition-modulated atomic layer stacking}

Finally, the reason for the excess theoretical limit of 0.25 for the corrected $I_{10.0} / I_{11.0}$, as shown in Fig. 4 , is discussed. According to Eq. (4), when the Co-Ir alloys have composition-modulated atomic layer with perfect hcp structure, normalized $F_{11.0}$ and $F_{10.0}$ by atomic scattering factor of each diffraction angle are given by:

$$
\begin{aligned}
F_{11.0} / f_{\mathrm{ave}}^{11.0} & =\sum_{j=1}^{N} f_{j}^{11.0} e^{-2 \pi i\left(u_{j}+v_{j}\right)} / f_{\mathrm{ave}}^{11.0} \\
& =\left\{f_{\mathrm{Co}}^{11.0}\left(N_{\mathrm{A}}^{\mathrm{Co}}+N_{\mathrm{B}}^{\mathrm{Co}}\right)+f_{\mathrm{Ir}}^{11.0}\left(N_{\mathrm{A}}^{\mathrm{Ir}}+N_{\mathrm{B}}^{\mathrm{Ir}}\right)\right\} / f_{\mathrm{ave}}^{11.0} \\
& =\left(N_{\mathrm{A}}^{\mathrm{Co}}+N_{\mathrm{B}}^{\mathrm{Co}}\right)+\left(N_{\mathrm{A}}^{\mathrm{Ir}}+N_{\mathrm{Br}}^{\mathrm{Ir}}\right)=N \\
F_{10.0} / f_{\mathrm{ave}}^{10.0} & =\sum_{j=1}^{N} f_{j}^{10.0} e^{-2 \pi u_{j}} / f_{\mathrm{ave}}^{10.0} \\
& =\left\{f_{\mathrm{Co}}^{10.0}\left(N_{\mathrm{A}}^{\mathrm{Co}}+N_{\mathrm{B}}^{\mathrm{Co}} e^{-2 \pi i \frac{1}{3}}\right)+f_{\mathrm{Ir}}^{10.0}\left(N_{\mathrm{A}}^{\mathrm{Ir}}+N_{\mathrm{B}}^{\mathrm{Ir}} e^{-2 \pi i \frac{1}{3}}\right)\right\} / f_{\mathrm{ave}}^{10.0} \\
& =\frac{f_{\mathrm{Co}}^{10.0}\left(N_{\mathrm{A}}^{\mathrm{Co}}+N_{\mathrm{B}}^{\mathrm{Co}} e^{-2 \pi i \frac{1}{3}}\right)+f_{\mathrm{Ir}}^{10.0}\left(N_{\mathrm{A}}^{\mathrm{Ir}}+N_{\mathrm{B}}^{\mathrm{Ir}} e^{-2 \pi i \frac{1}{3}}\right)}{f_{\mathrm{Co}}^{10.0}\left(N_{\mathrm{A}}^{\mathrm{Co}}+N_{\mathrm{B}}^{\mathrm{Co}}\right)+f_{\mathrm{Co}}^{10.0}\left(N_{\mathrm{A}}^{\mathrm{Ir}}+N_{\mathrm{Br}}^{\mathrm{Ir}}\right)} N .
\end{aligned}
$$

Therefore, the value of (corrected $I_{10.0} / I_{11.0}-0.25$ ) can be expressed as:

$$
\begin{aligned}
& \text { Corrected } I_{10.0} / I_{11.0}-\frac{1}{4} \\
& =\left(\frac{f_{\text {ave }}^{11.0}}{f_{\text {ave }}^{10.0}}\right)^{2}\left|\frac{F_{10.0}}{F_{11.0}}\right|^{2}-\frac{1}{4} \\
& =\left|\frac{f_{\mathrm{Co}}^{10.0}\left(N_{\mathrm{A}}^{\mathrm{Co}}+N_{\mathrm{B}}^{\mathrm{Co}} e^{-2 \pi \frac{1}{3}}\right)+f_{\mathrm{Ir}}^{10.0}\left(N_{\mathrm{A}}^{\mathrm{Ir}}+N_{\mathrm{B}}^{\mathrm{Ir}} e^{-2 \pi i \frac{1}{3}}\right)}{f_{\mathrm{Co}}^{10.0}\left(N_{\mathrm{A}}^{\mathrm{Co}}+N_{\mathrm{B}}^{\mathrm{Co}}\right)+f_{\mathrm{Ir}}^{10.0}\left(N_{\mathrm{A}}^{\mathrm{Ir}}+N_{\mathrm{B}}^{\mathrm{Ir}}\right)}\right|-\frac{1}{4} \\
& =\frac{3}{4}\left\{\frac{\left(f_{\mathrm{Co}}^{10.0} N_{\mathrm{A}}^{\mathrm{Co}}+f_{\mathrm{lr}}^{10.0} N_{\mathrm{A}}^{\mathrm{Ir}}\right)-\left(f_{\mathrm{Co}}^{10.0} N_{\mathrm{B}}^{\mathrm{Co}}+f_{\mathrm{r}}^{10.0} N_{\mathrm{B}}^{\mathrm{Ir}}\right)}{\left(f_{\mathrm{Co}}^{10.0} N_{\mathrm{A}}^{\mathrm{Co}}+f_{\mathrm{Ir}}^{10.0} N_{\mathrm{A}}^{\mathrm{rr}}\right)+\left(f_{\mathrm{Co}}^{10.0} N_{\mathrm{B}}^{\mathrm{Co}}+f_{\mathrm{lr}}^{10.0} N_{\mathrm{B}}^{\mathrm{Ir}}\right)}\right\}^{2} \geq 0 .
\end{aligned}
$$

In this case, the atomic scattering factors $f_{\mathrm{Co}^{1}}{ }^{11.0}$ and $f_{\mathrm{rr}}{ }^{11.0}$ in the corrected $I_{10.0} / I_{11.0}$ remain even normalization by atomic scattering factor was adopted; thus, information of scattering factors are reflected in the corrected $I_{10.0} / I_{11.0}$. In conclusion, composition modulation is the reason that the value of the corrected $I_{10.0} / I_{11.0}$ exceeded the theoretical limitation of 0.25 as shown in Fig. 4.

We consider that clarifying the reason for the formation of this composition-modulated atomic layer structure in the alloy films with limited composition range is one of the issues to be resolved in future work.

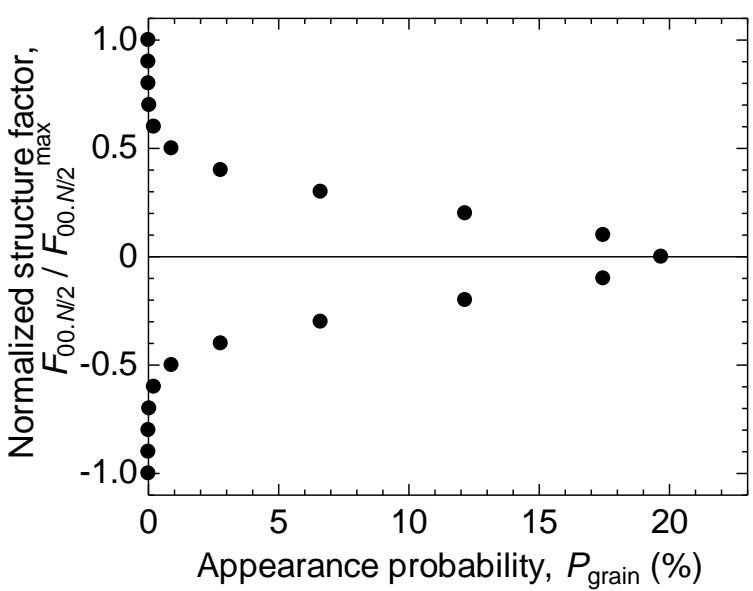

Fig. 10 The structure factor $F_{00 . N / 2}$ normalized by the maximum $F_{00 . N 2}$ as a function of $P_{\text {grain }}$.

Acknowledgements The authors gratefully acknowledge Y. Nakamura and T. Uruno from the JX Nippon Mining \& Metals Co., Ltd. for their cooperation for the fabrication of the $\mathrm{Co}_{80} \mathrm{Ir}_{20}$ target. The authors would also like to thank K. Yamaguchi and N. Goto from Ohara Inc. for providing the crystallized glass substrates. This work was supported in part by the Storage Research Consortium (SRC) of Japan and JSPS KAKENHI Grant Number $22 \cdot 2544$.

\section{References}

1) A. Hashimoto, S. Saito, and M. Takahashi: J. Appl. Phys., 99, 08Q907 (2006).

2) A. Hashimoto, S. Saito, and M. Takahashi: J. Magn. Soc. Jpn., 30, 135 (2006).

3) M. Takahashi and S. Saito: J. Magn. Magn. Mater., 320, 2868 (2008).

4) M. Takahashi, M. Tsunoda, and S. Saito: J. Magn. Magn. Mater., 321, 539 (2009).

5) K. Yoshida, M. Yokoe, Y. Ishikawa, and Y. Kanai: IEEE Trans. Magn., 46, 2466 (2010).

6) M. Igarashi, Y. Suzuki, and Y. Sato: IEEE Trans. Magn., 46, 3738 (2010).

7) M. Takahashi, Y. Takahashi, and H. Shoji: IEEE Trans. Magn., 37, 2179 (2001).

8) M. Takahashi and S. Kadowaki: J. Phys. Soc. Jpn., 48, 1391 (1980).

9) T. Okita and Y. Makino: J. Phys. Soc. Jpn., 25, 120 (1968).

10) N. Kikuchi, O. Kitakami, S. Okamoto, Y. Shimada, A. Sakuma, Y. Otani, and K. Fukamichi: J. Phys. Condens. Matter., 11, L485 (1999).

11) D. Hasegawa, S. Nakasaka, M. Sato, T. Ogawa, and M. Takahashi: IEEE Tran. Magn., 42, 2085 (2006).

12) N. Nozawa, S. Saito, S. Hinata, and M. Takahashi: IEICE Tech. Report, MR2012-16, 41 (2012).

13) Y. Yamada, T. Suzuki, and E. N. Abarra: IEEE Trans. Magn, 33, 3622 (1997). 
14) N. Nozawa, S. Saito, T. Kimura, K. Shibuya, K. Hoshino, S. Hinata, and M. Takahashi: Appl. Phys. Lett., 102, 012407 (2013).

15) S. Saito, A. Hashimoto, D. Hasegawa, and M. Migaku: J. Phys. D: Appl. Phys., 42, 145007 (2009).

16) A. Hashimoto, S. Saito, N. Itagaki, and M. Takahashi: Appl. Phys. Lett., 89, 262508 (2006).

17) A. Hashimoto, S. Saito, N. Itagaki, and M. Takahashi: J. Phys. D: Appl. Phys., 41, 012002 (2008)

18) S. J. Pennycook: Ultramicroscopy, 30, 58 (1989).
19) P. A. Midgley and M. Weyland: Ultramicroscopy, 96, 413 (2003).

20) H. Sato, T. Shimatsu, Y. Okazaki, H. Muraoka, and H. Aoi: J. Appl. Phys., 103, 07E114 (2008).

21) A.-C. Sun, F.-T. Yuan, J.-H. Hsu, and H. Y. Lee: Scripta Materialia, 61, 713 (2009).

22) Warren B. E.: X-ray Diffraction (Reading, MA: Addison -Wesley), 284 (1968)

Received Oct. 19, 2012; Revised Jan. 17, 2013; Accepted Feb. 06, 2013 